\title{
Spin physics with CLAS and CLAS12
}

Angela Biselli

Fairfield University, abiselli@fairfield.edu

Follow this and additional works at: https://digitalcommons.fairfield.edu/physics-facultypubs Copyright 2011 AIP Publishing

The final publisher PDF has been archived here with permission from the copyright holder. This article may be downloaded for personal use only. Any other use requires prior permission of the author and AIP Publishing. The following article appeared in A. Biselli, "Spin physics with CLAS and CLAS12," AIP Conf. Proc. 1388, 53 (2011), proceedings of the conference Baryons 2010, Osaka, Japan, 7-11 December 2010.and may be found at http://aip.scitation.org/doi/abs/ 10.1063/1.3647350.

\section{Repository Citation}

Biselli, Angela, "Spin physics with CLAS and CLAS12" (2011). Physics Faculty Publications. 128.

https://digitalcommons.fairfield.edu/physics-facultypubs/128

\section{Published Citation}

Biselli, A. (2011, October). Spin physics with CLAS and CLAS12. In AIP Conference Proceedings Vol. 1388, No. 1, pp. 53-59.

This item has been accepted for inclusion in DigitalCommons@Fairfield by an authorized administrator of DigitalCommons@Fairfield. It is brought to you by DigitalCommons@Fairfield with permission from the rightsholder(s) and is protected by copyright and/or related rights. You are free to use this item in any way that is permitted by the copyright and related rights legislation that applies to your use. For other uses, you need to obtain permission from the rights-holder(s) directly, unless additional rights are indicated by a Creative Commons license in the record and/or on the work itself. For more information, please contact digitalcommons@fairfield.edu. 


\section{Spin physics with CLAS and CLAS12}

Angela Biselli

Citation: AIP Conference Proceedings 1388, 53 (2011);

View online: https://doi.org/10.1063/1.3647350

View Table of Contents: http://aip.scitation.org/toc/apc/1388/1

Published by the American Institute of Physics 


\title{
Spin physics with CLAS and CLAS12
}

\author{
Angela Biselli
}

Fairfield University, Fairfield, CT 06828

\begin{abstract}
An extensive experimental program to measure the spin structure of the nucleon has been conducted in Hall B at Jefferson Lab with the CEBAF Large Acceptance Spectrometer (CLAS) in the last decade. Using a longitudinally polarized beam scattering off longitudinally polarized $\mathrm{NH}_{3}$ and $\mathrm{ND}_{3}$ targets, inclusive Deep Inelastic Scattering (DIS), Semi-Inclusive DIS (SIDIS) and DVCS experiments were carried out that make a significant contributions to the existing data. The inclusive double spin asymmetry $A_{\|}$was measured over a large range in $Q^{2}$ and $W$, providing data of impressively high precision that give a better understanding of the structure of the nucleon in the DIS and the valence quarks regions. Using parameterizations $A_{2}$ and $F_{1}$ from world data, the virtual photon asymmetry $A_{1}$ and the structure function $g_{1}$ were extracted in a $Q^{2}$ range from 0.05 to $5 \mathrm{GeV}^{2}$ and a $W$ range from 1.08 to $3.0 \mathrm{GeV}$. As a result of the extended kinematical range, first moments of structure functions were measured over a large range in $Q^{2}$ and duality was tested. Furthermore, newly proposed experiments, using an upgraded accelerator at Jefferson Laboratory and an improved CLAS detector (CLAS12), are expected to increase the statistical precision of the current measurements and extend them to kinematic regions presently not accessible, such as high $x$. This will improve significantly our knowledge of the structure of the nucleon, including parton distribution functions, duality and higher twists contributions.
\end{abstract}

Keywords: Spin structure, Nucleon Spin

PACS: $13.60 . \mathrm{Hb}, 13.88 .+\mathrm{e}, 14.20 . \mathrm{Dh}$

\section{INTRODUCTION}

In the last 30 years, the spin structure of the nucleon has been the focus of many experimental programs. Inclusive inelastic scattering of polarized electrons off polarized nucleons is a powerful tool to study the polarized structure functions and their moments. For a longitudinally polarized beam and target the measured asymmetry is $A_{\|}=\frac{\sigma^{+}-\sigma^{-}}{\sigma^{+}+\sigma^{-}}$ where $\sigma^{+}$and $\sigma^{-}$are the cross sections for beam and target polarizations oriented antiparallel and parallel, respectively. $A_{\|}$is related to the virtual photon asymmetries $A_{1}$ and $A_{2}$ by

$$
\frac{A_{\|}}{D}=A_{1}+\eta A_{2}
$$

where $\eta=\varepsilon Q /\left(E-E^{\prime} \varepsilon\right), D=\left(1-E^{\prime} \varepsilon / E\right) /(1+\varepsilon R)$ is the photon depolarization factor, $R=\sigma_{L} / \sigma_{T}$ is the ratio of longitudinal and transverse virtual photon-absorption cross sections, $Q^{2}$ is the squared 4-momentum transfer, and $\varepsilon=\left(1+2 \frac{\vec{q}^{2}}{Q^{2}} \tan ^{2} \frac{\theta_{e}}{2}\right)^{-1}$ is the degree of transverse virtual photon polarization. The asymmetry $A_{1}$ is related to the difference of virtual photo-absorption cross sections for total helicity between photon and nucleon of $1 / 2$ and $3 / 2 . A_{2}$ is related to the interference term between the transverse and longitudinal photon-nucleon amplitudes.

International Conference on the Structure of Baryons

AIP Conf. Proc. 1388, 53-59 (2011); doi: 10.1063/1.3647350

(C) 2011 American Institute of Physics 978-0-7354-0952-1/\$30.00 
Given the virtual photon asymmetries, one can extract to the spin structure function $g_{1}$ using:

$$
g_{1}\left(x, Q^{2}\right)=\frac{\tau}{1+\tau}\left(A_{1}+\frac{1}{\sqrt{\tau}} A_{2}\right) F_{1}\left(x, Q^{2}\right)
$$

where $\tau=v^{2} / Q^{2}, v$ is the energy transfer of the virtual photon, and $F_{1}$ is the unpolarized structure function. These quantities can be used to test sum rules, e.g. the GerasimovDrell-Hearn sum rule, to test duality and to extract parton distribution functions. An extensive review of experimental results can be found in [1].

Although without a doubt inclusive scattering measurements give a rich picture of the spin structure of the nucleon, they do not explore its three dimensional properties. In recent years experimental programs have been devoted to Semi-Inclusive DIS (SIDIS) and hard exclusive processes such as DVCS in order to achieve a full three dimensional picture of the nucleon. SIDIS experiments detect a hadron in the final state besides the scattered electron, gaining sensitivity to new information such as the transverse momenta dependent parton distributions (TMDs) or the flavor of the struck quark. DVCS processes, where a virtual photon is absorbed by the nucleon and a hadron plus a real photon are emitted, allow access to Generalized Parton Distributions (GPD) which are fully correlated quark distributions in both coordinate and momentum space. The DVCS amplitude depends on four GPDs: $H, \tilde{H}, E$, and $\tilde{E}$, which are functions of the momentum fraction of the struck quark, $x$, the longitudinal momentum fraction transferred to the proton, $\xi=x_{B} /\left(2-x_{B}\right)$, and the momentum transfer, $t$, between virtual and real photons. These four GPDs are related to both the spacial distributions (elastic form factors) and the momentum density (parton distributions). Experimentally the DVCS signal can be enhanced by measuring asymmetries which are proportional to the interference between the DVCS process $\left(T_{\mathrm{DVCS}}\right)$ and the dominant Bethe-Heitler process $\left(T_{\mathrm{BH}}\right)$.

$$
A=\frac{\sigma^{+}-\sigma^{-}}{\sigma^{+}+\sigma^{-}} \propto \frac{I}{\left|T_{\mathrm{DVCS}}\right|^{2}+\left|T_{\mathrm{BH}}\right|^{2}+I}
$$

where $I=T_{\mathrm{DVCS}} T_{\mathrm{BH}}^{*}+T_{\mathrm{DVCS}}^{*} T_{\mathrm{BH}}$. Beam, target and double spin asymmetries have different sensitivities to the GPDs and only a measurement of all the asymmetries over a large kinematic range will allow a complete extraction of the GPDs.

\section{EXPERIMENTS}

\section{CLAS experiments}

The two key experiments devoted to the measurement of spin structure functions in Hall-B at Jefferson Laboratory are the EG1 experiment, run in two separate periods in 1998 and 2000 and the EG4 experiment, run in 2006. The Continuous Electron Beam Accelerator Facility (CEBAF) delivered a longitudinally polarized electron beam with energies of 1.6, 2.5, 4.2, and 5.75 GeV for EG1 and 1.1, 1.3, 1.5, 2.2, and 3.0 GeV for EG4. Hall-B hosts the CEBAF Large Acceptance Spectrometer (CLAS), which allows multiple-particle identification with a nearly $2 \pi$ acceptance. The beam was scattered 
off longitudinally polarized solid, 1-cm-long, ammonia targets, ${ }^{15} \mathrm{NH}_{3}$ and ${ }^{15} \mathrm{ND}_{3}$, polarized up to $70 \%$ and $40 \%$, respectively, via Dynamic Nuclear Polarization [2]. Additional ${ }^{12} C,{ }^{15} N$, and the empty target cells were used for background studies. The trigger used was a coincidence signal between the Cherenkov counters and the electromagnetic calorimeters, which allowed the identification of electrons. Protons, deflected by a superconducting toroidal field, are detected by three layers of drift chambers for momentum identification and scintillator paddles for time-of-flight identification. The EG1 experiment achieved a kinematic coverage from 0.05 up to $5 \mathrm{GeV}^{2}$ in $Q^{2}$ and from 1.08 to 3 $\mathrm{GeV}$ in $W$. The EG4 experiment utilized an additional Cherenkov counter in one sector to detect electrons with angles as low as $6^{\circ}$ and therefore to extend the $Q^{2}$ range down to $0.015 \mathrm{GeV}^{2}$.

The key experiment for SIDIS and DVCS studies with the polarized target is the EG1-DVCS experiment which ran in 2009. While pioneering measurements were done using the EG1 data[3, 4], EG1-DVCS was the first dedicated DVCS experiment with the longitudinally polarized target at Jefferson Lab. The experimental setup was identical to the EG1 experiment with an additional Internal Calorimeter (IC) for neutral particles detection at small angles from $4-15^{\circ}$. Neutral particles with angles from $17^{\circ}$ to $43^{\circ}$ are detected by the standard electromagnetic calorimeter. The IC consists of 424 lead tungstate $\left(\mathrm{PbWO}_{4}\right)$ crystals, $16 \mathrm{~cm}$ in length (18 radiation lengths) with an energy and angle resolution of $4.5 \%$ and $4 \mathrm{mrad}$, respectively. The large acceptance of the CLAS detector in conjunction with the IC allows complete detection of the three final state particles from the reaction ep $\rightarrow$ ep $\gamma$ over a large kinematic range. Therefore, kinematic cuts can drastically reduce the nuclear background from ${ }^{14} \mathrm{~N}$. Contamination from $\pi^{0}$ is corrected by measuring the ep $\pi^{0}$ asymmetry and by estimating the amount of contamination using Monte Carlo simulations. The experiment ran for 109 days at beam energies of $5.9 \mathrm{GeV}$, giving an accessible range in $Q^{2}$ from 1 to $4 \mathrm{GeV}^{2}$, and in $x_{B}$ from 0.15 to 0.55 .

\section{CLAS12 experiments}

The upgrade of the accelerator at Jefferson Laboratory will increase the maximum beam energy to $12 \mathrm{GeV}$ and several experiments have been proposed to enhance and extend the spin program in Hall B. The proposed experiments will use the standard equipment of the upgraded CLAS12 detector. Similar to its predecessor, CLAS12 will have six sectors covering a nearly $2 \pi$ angle. Each sector, delimited by superconducting coils generating a toroidal field, will be equipped with drift chambers for tracking, Cherenkov counters for trigger and particle identification, time-of-flight for particle identification and electromagnetic calorimeters as a trigger and neutral particles detector. In addition to the six sectors for forward-going particles, CLAS12 will be equipped with a central detector to detect slow particles scattering at large angles. The central detector will have a have a silicon vertex detector, a time of flight system and a solenoid which will simultaneously provide the magnetic field for the tracking and for the polarized target. The polarized target will be a solid state target polarized via DNP as in the EG1 experiment. 

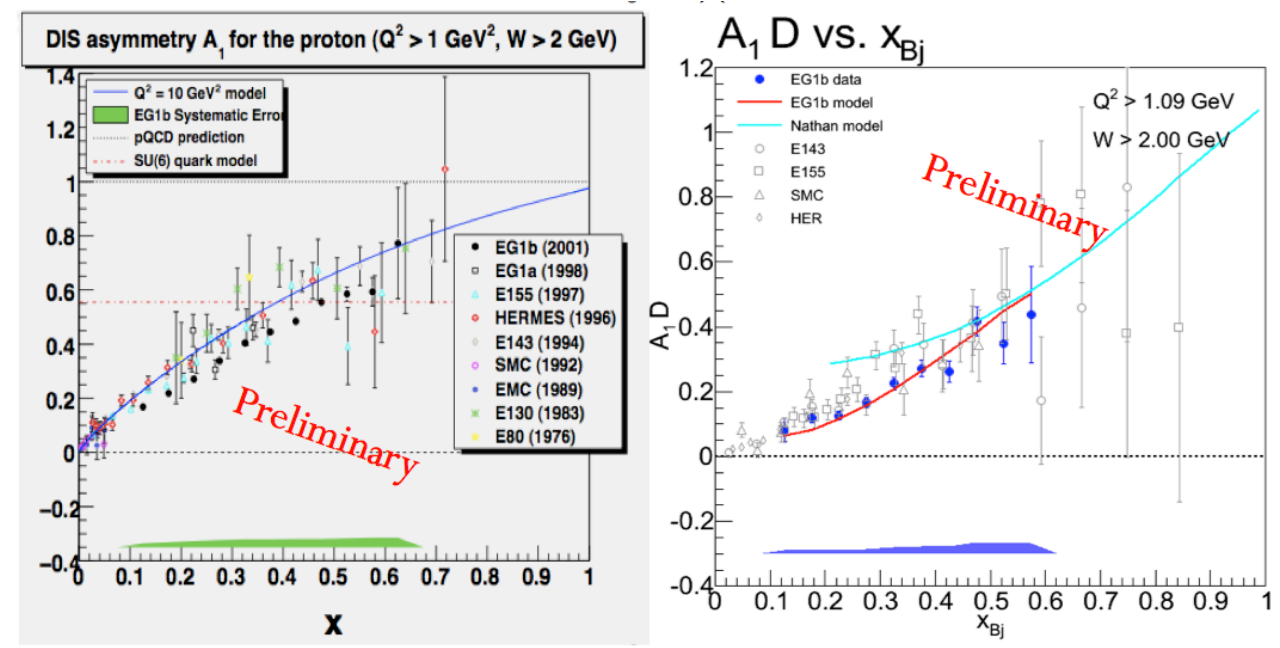

FIGURE 1. Virtual photon asymmetry $A_{1}$ vs. $\mathrm{x}$ for proton (left) and deuteron (right).

Experiments to study both spin structure functions and generalized parton distributions are planned. The increased beam energy of $11 \mathrm{GeV}$ will allow one to explore previously inaccessible kinematic regions, such as $x$ up to 0.8 and high $Q^{2}$. The new data will dramatically improve the statistical precision of the current measurements, improving the extraction of quantities such as parton distributions and generalized parton distributions.

\section{SELECTED RESULTS}

A quantity of great interest is the virtual photon asymmetry $A_{1}$, in particular its behavior at high $x$, where $\mathrm{SU}(6), \mathrm{pQCD}$ and the hyperfine perturbed quark model give different predictions. Using a model for $A_{2}$, the virtual photon asymmetry $A_{1}$ was extracted using the EG1 data, both for the proton and the deuteron. Figure 1 shows preliminary results for the analysis of the complete EG1 data set, averaged in the deep inelastic region $Q^{2}>$ $1 \mathrm{GeV}^{2}$ and $W>2 \mathrm{GeV}$. The results confirm the trend of $A_{1}$ to exceed the SU(6) limit at $x=1$, which is $5 / 9$ for the proton and $1 / 3$ for the deuteron already reported in [5]. Data are consistent with the hyperfine perturbed quark model at higher $x$ (not shown in the figure), where the valence quarks are expected to dominate.

Using the parametrization of world data for $F_{1}$ and the $A_{1}$ data, the structure function $g_{1}$ was extracted over a large kinematic range both in the resonance and DIS region. Figure 2 shows the results for the proton. The structure function $g_{1}$ is driven negative at high $x$ by the $\Delta(1232)$ resonance at low $Q^{2}$ and becomes positive everywhere at high $Q^{2}$ where it is in agreement with the DIS curve at $Q^{2}=10 \mathrm{GeV}^{2}$.

The large $x$-range for a fixed $Q^{2}$ interval, allows us to integrate over $x$ and therefore to extract moments of the spin structure functions. Figure 3 shows the first moment of the structure function $g_{1}$ for both proton and deuteron. The world data parameterization was used to include the unmeasured part to the integral down to $x=0.001$, however only the $Q^{2}$ bins where the measured contribution to the integral constitutes at least $50 \%$ are 

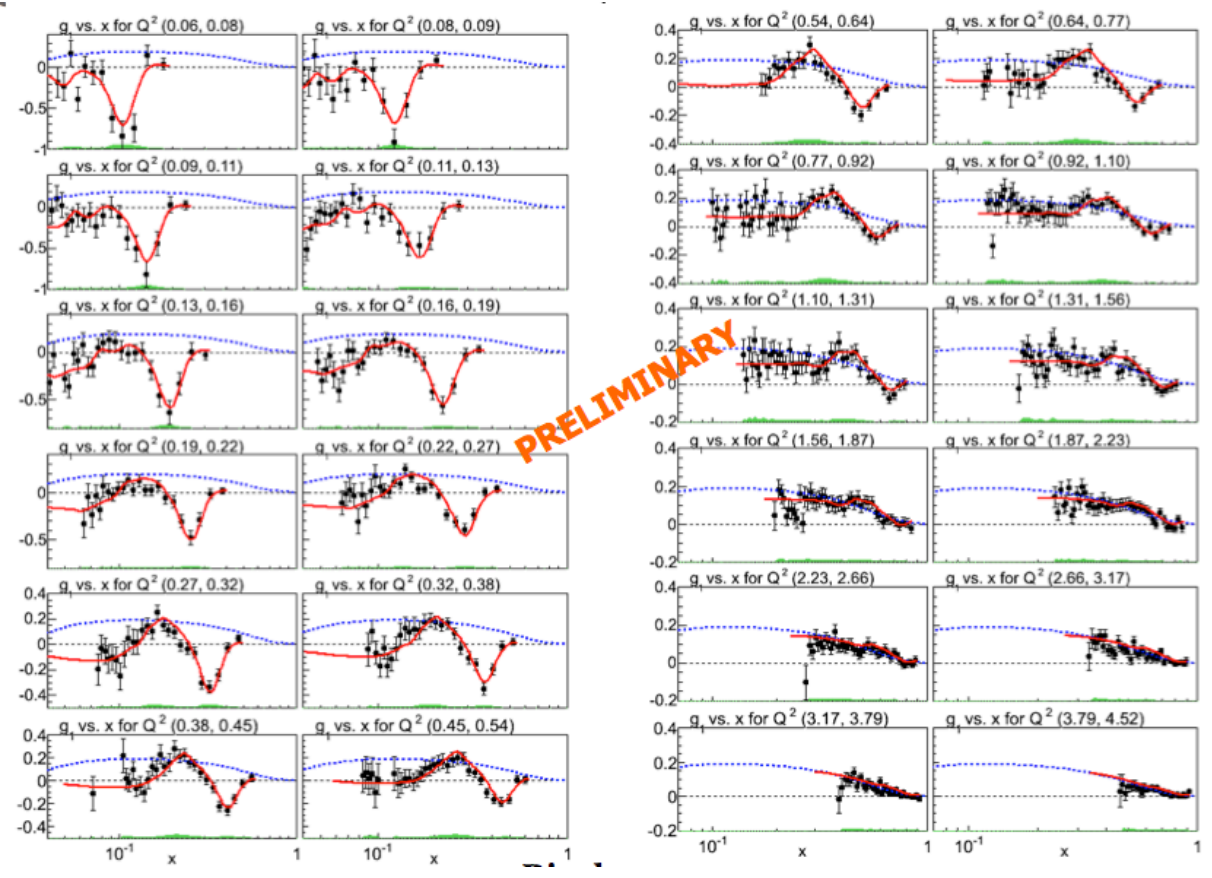

FIGURE 2. $g_{1}$ vs $x$ for several $Q^{2}$ bins from 0.06 to $2.23 \mathrm{GeV}^{2}$ for the proton.

shown. The low $Q^{2}$ data points are consistent with the $I_{G D H}$ limit, and this agreement will be tested even further by the EG4 experiment, which will extend down to $Q^{2}=0.015$ $\mathrm{GeV}^{2}$ and increase the precision of the existing data points. The $Q^{2}$ evolution is in fairly good agreement with the phenomenological model by Burkert and Ioffe [6], which parameterizes the transition between the real photon point and the DIS regime using measured pion electro- and photo-production resonance amplitudes. The comparison of data at low $Q^{2}$ with the ChPT calculation of Ji (dashed curve) [7] shows a disagreement; however a fit of the data using a polynomial in $Q^{2}$ up to $Q^{8}$ shows a non negligible $Q^{6}$ term even at $Q^{2}<0.1 \mathrm{GeV}^{2}$, while Ji's calculation is an expansion only up to $Q^{4}$.

Structure functions and virtual photon asymmetries can be used to extract parton distribution functions. The left panel of Figure 4 shows the result for the up and down quark distributions from a fit of the EG1 data combined with Hall A, SLAC, and semiinclusive HERMES data [8]. The results show a disagreement, especially for the d quark with the pQCD expectations (solid line) but it is in better agreement with pQCD when the effects of orbital angular momentum are taken into account (dashed curve). The right panel shows the error bands for the quark distributions in the fit by Leader, Stamenov and Siderov (LSS'05) [9]. The curves indicate the impact of the EG1 and COMPASS data on the LSS'05 analysis. The CLAS12 data are expected to significantly reduce the errors, especially at high $x$, and it will be possible to conclusively test the effect of orbital angular momentum.

In addition to the parton distributions one can study GPDs and TMDs. The analyses of the EG1-DVCS experiment are at too early a stage for the extraction of these quantities, however, single and double spin asymmetries have been extracted, showing promising results. The right panel of Figure 5 shows the target asymmetry for the $\pi^{+}$SIDIS 

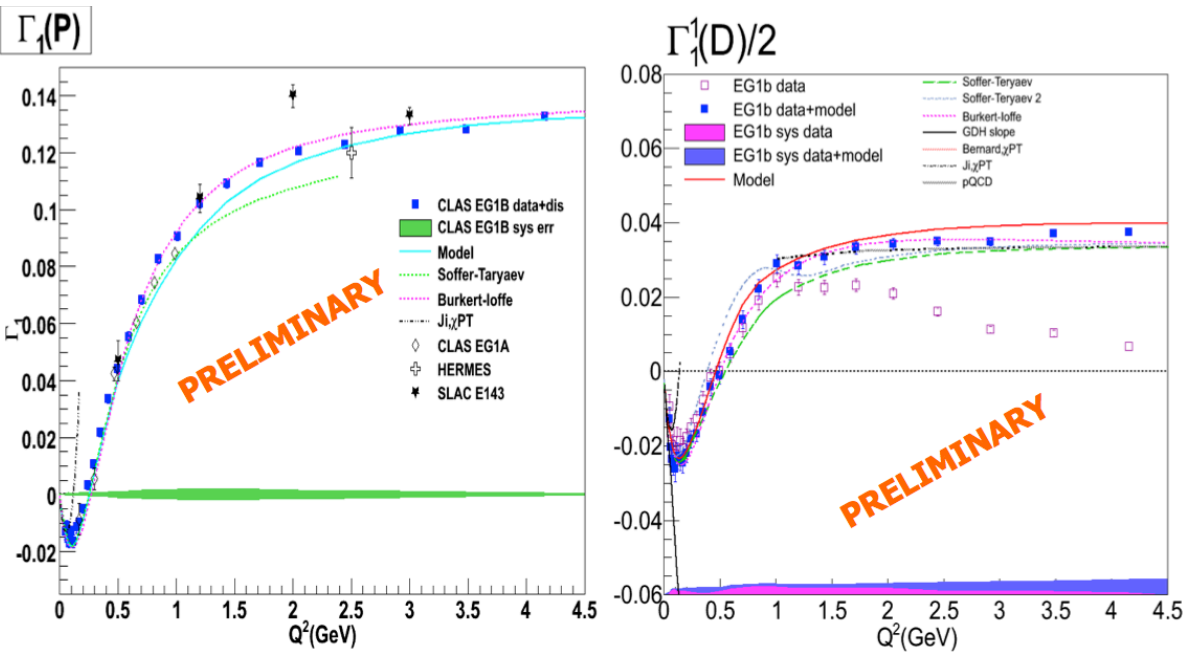

FIGURE 3. $\quad \Gamma_{p}^{1}$ vs $Q^{2}$ for the proton (left) and deuteron (right). The Figure shows the phenomenological predictions (Burkert-Ioffe, Soffer- Teryaev, ChPT) and the GDH slope.
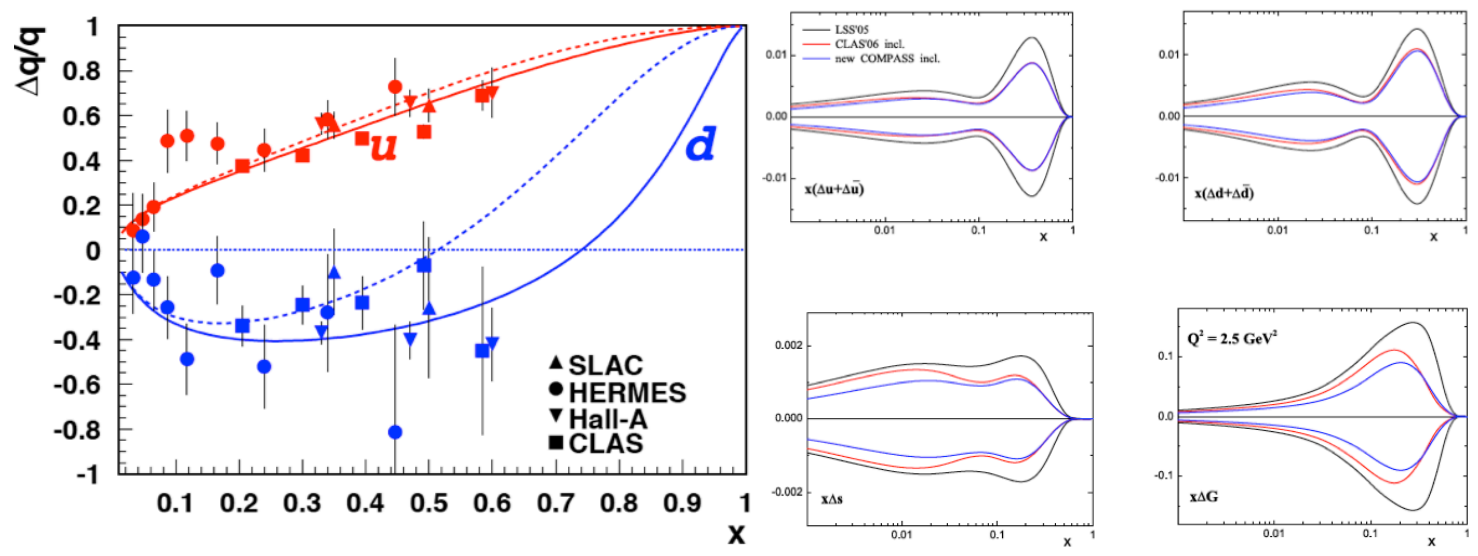

FIGURE 4. Left: $\Delta u / u$ and $\Delta d / d$ extracted from Halls A and B, SLAC, and HERMES data compared with the pQCD expectations without (solid) and with (dashed) effects of orbital angular momentum. Right: Uncertainty on polarized parton distributions in the LSS'05 fit before (outer-most) and after (middle and inner-most line) the inclusion of Jefferson Lab and COMPASS data.

channel and the right panel show the the target DVCS asymmetry. Both asymmetries are consistent with the expected $\phi$ dependence and show a great improvement in statistics as compared to the previous measurements done with the EG1 data.

In summary, the analyses of the full data set of the EG1 run for both the proton and deuteron targets give measurements with unprecedented precision of $A_{1}, g_{1}$, and related quantities over a large kinematic range. The DVCS and SIDIS experiments will provide new constraints on GPDs and TMDs. Finally experiments with the upcoming upgrade, CLAS12, will greatly increase the statistical precision and the kinematic coverage of existing data and will give a fuller picture of the spin structure of the nucleon. 

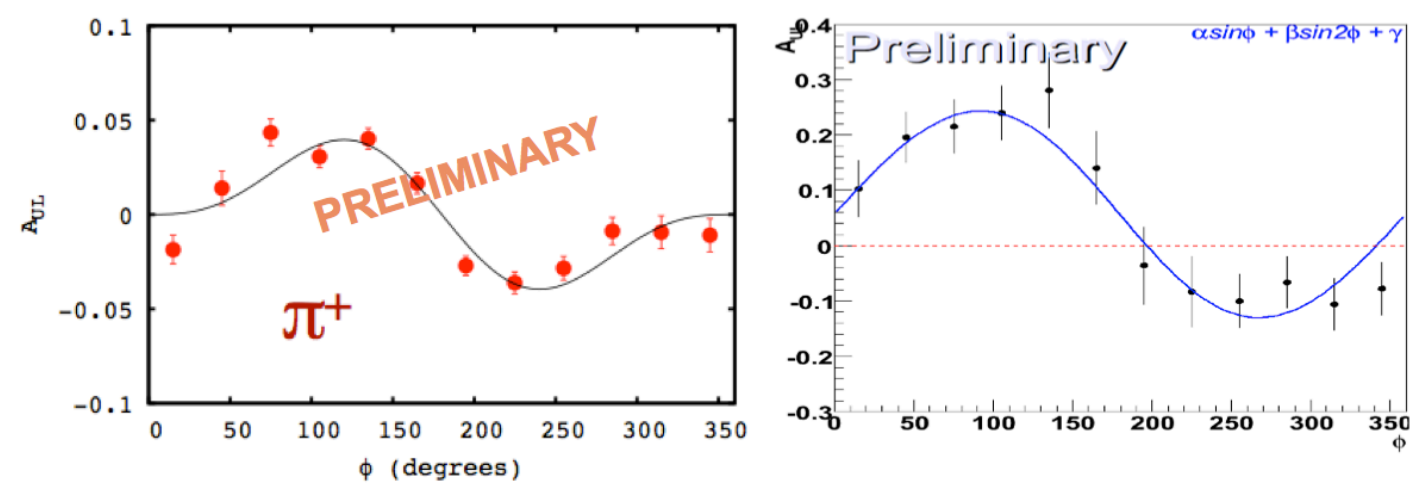

FIGURE 5. Preliminary target asymmetries for the DVCS channel (left) and the SIDIS $\pi^{+}$channel (right)

\section{ACKNOWLEDGMENTS}

We would like to thank the staff of the Accelerator and Physics Divisions at the Jefferson Lab for their outstanding efforts to provide us with the high quality beam and the facilities for data analysis. This work was supported by the U.S. Department of Energy and the National Science Foundation, the French Commissariat a l'Energie Atomique, the Italian Istituto Nazionale di Fisica Nucleare, and the Korean Science and Engineering Foundation. Jefferson Science Associates (JSA) operates the Thomas Jefferson National Accelerator Facility for the United States Department of Energy under contract DEAC05- 84ER40150.

\section{REFERENCES}

1. S. E. Kuhn, J. P. Chen, and E. Leader, Prog. Part. Nucl. Phys. 63, 1-50 (2009).

2. C. D. Keith et al., Nucl. Instrum. Meth. A 501, 327 (2003).

3. H. Avakian et al., Phys. Rev. Lett., 105, 262002 (2010).

4. S. Chen et al., Phys. Rev. Lett., 97, 072002 (2006).

5. K. V. Dharmawardane et al., Phys. Lett. B 641, 11 (2006).

6. V. D. Burkert and B. L. Ioffe, Phys. Lett. B 296, 223 (1992).

7. X. D. Ji, C. W. Kao and J. Osborne, Phys. Lett. B 472, 1 (2000).

8. H. Avakian, S. J. Brodsky, A. Deur, and F. Yuan, Phys. Rev. Lett., 99,082001 (2007).

9. E. Leader, A. V. Sidorov, and D. B. Stamenov, Phys. Rev., D75, 074027 (2007). 